\title{
Methode zur Isolierung von Antikörpern vom IgM-Typusi)
}

\author{
Von K. SCHUMACHER \\ Aus dem Physiologisch-Chemischen Institut der Universität Würzburg (Direktor: Prof. Dr. F. Turbaf)
}

(Eingegangen am 15. Dezember 1965)

Es wird eine Methode zur schonenden Isolierung von Antikörpern vom IgM-Typus beschrieben. Die Isolierung erfolgte über mehrere Trennschritte: Zonenelektrophorese, Gelfiltration und Ultrazentrifugation im Saccharosegradienten. Die biologische Aktivität des Antikörpers, in diesem Fall eines Autoantikörpers gegen weiße Hirnsubstanz aus Seren von Patienten mit Multipler Sklerose, blieb erhalten.

A method is described for the protective isolation of antibodies of the IgM type. The isolation takes place in several separatory stages: zone electrophoresis, gel filtration and ultracentrifugation in a sucrose gradient. The biological activity of the antibody, in this case an antibody against white brain matter from the sera of patients with multiple sclerosis, remains unchanged.

Sämtliche im Serum auftretenden Antikörper gehören zu den Gamma- oder Immunglobulinen. Ihren verschiedenen Wanderungsgeschwindigkeiten im elektrischen Feld entsprechend unterscheiden wir drei große Gruppen von Immunglobulinen, die $\gamma-2, \gamma-1 \mathrm{~A}$ und $\gamma$-1-M Globuline, wobei jede dieser Gruppen in sich nicht einheitlich ist, sondern mehr oder minder zahlreiche Antikörper verschiedener Spezifität enthält. Nach einer in jüngster Zeit getroffenen Vereinbarung2) (1) werden die Immunglobuline als IgG, IgA und IgM Globuline bezeichnet. Dazu kommt eine nur in Spuren im Serum vorhandene Gruppe von Immunglobulinen, die die Bezeichnung IgD erhalten hat (2).

Zur Auftrennung eines Eiweißgemisches wie es Serum oder Plasma darstellen, nutzt man die Unterschiede der Proteine in bezug auf isoelektrischen Punkt, Teilchengröße, Dichte, Löslichkeit und Hydratisierbarkeit aus (3). Dabei gilt generell, besonders aber für die Isolierung von Antikörpern, deren spezifische Aktivität an die Intaktheit ihrer Tertiärstruktur gebunden ist, da $\beta$ nur wirklich schonende Trennverfahren Verwendung finden dürfen. Vor allem Antikörper vom IgM-Typus erweisen sich mit zunehmender Reinigung als sehr labil und leicht denaturierbar. Zur Isolierung von Gamma-Makroglobulinen kommen deshalb nur wenige Trennverfahren in Frage, u. a. Euglobulinpräzipitation, Zonenelektrophorese, Gelfiltration, Chromatographie an Ionenaustauschern, Ultrazentrifugation und Präzipitation mit Salzen unter milden Bedingungen (3-8). Die Auswahl einer Trennmethode geschieht dabei vor allem nach der Schnelligkeit des Verfahrens und der gewünschten Reinheit des Präparates.

Im Rahmen unserer gemeinsam mit J. Ross durchgeführten Untersuchungen (9) zur Charakterisierung des bei Patienten mit Multipler Sklerose im Serum auftretenden Autoimmunantikörpers gegen weiße Hirnsubstanz wurde die Isolierung dieses Antikörpers notwendig. Vorversuche mit der Fraktionierung antikörperhaltiger Seren durch Gelfiltration an Sephadex G-200 hatten ergeben, daß sich die Antikörperaktivität ausschließlich in der ersten (19 S) Fraktion befand. Es war demnach wahrscheinlich, daß es sich bei dem gesuchten Anti-

\footnotetext{
1) Mit Unterstützung der Deutschen Forschungsgemeinschaft.

2) $\mathrm{IgG}=\gamma$-G $=\gamma-2=\gamma$-ss $=7 \mathrm{~S} \gamma$-Globulin.

$\operatorname{IgA}=\gamma-\mathrm{A}=\gamma-1-\mathrm{A}=\beta-2 \mathrm{~A}-\mathrm{Globulin}$.

$\operatorname{IgM}=\gamma-\mathrm{M}=\gamma-1-\mathrm{M}=\beta-2 \mathrm{M}-\mathrm{Globulin}$.

IgD $=\gamma$-D-Globulin.
}

körper um ein Gamma-Globulin von IgM-Typus handelte. Eine Reindarstellung gelang aber allein mit Hilfe der Gelfiltration nicht, denn die erste Fraktion enthielt neben dem IgM Globulin auch $\beta$ - und $\alpha$-2-Lipoprotein und $\alpha$-2-Makroglobulin. Es wurden deshalb drei Trennverfabren miteinander kombiniert, Zonenelektrophorese, Gelfiltration und Ultrazentrifugation im Saccharosegradienten.

\section{Methodik}

Als Ausgangsmaterial dienten Seren von Patienten mit Multipler Sklerose, deren Antikörperaktivität zuvor im Doppeldiffusionstest nach OUCHTERLONY nachgewiesen worden war.

Zonenelektrophorese: Gerät Beckman C P. Nach ausgiebiger Dialyse des Serums gegen den Elektrophoresepuffer $(0,025 \mathrm{M}$ Na-BoratPuffer, $\mathrm{pH} 8,6$ ) wurden $30 \mathrm{~m} l$ Serum in das Vorratsgefä $\beta$ des Getätes (Beckman C P) gegeben und kontinuierlich innerhalb 24 Stdn. auf den gut äquilibrierten Papierbogen aufgetragen. Die elektrophoretische Trennung erfolgte bei konstanter Stromstärke von $90 \mathrm{~mA}$ mit einer Spannung von $580-620 \mathrm{~V}$.

Gelfiltration: Sephadex G-200 (Fa. Pharmacia Uppsala) gut in Puffer gewaschen, Säule $110 \times 4 \mathrm{~cm}$. Elutionspuffer: $0,1 \mathrm{~m}$ Tris-HCl, pH 8,0+0,2M NaCl. Elutionsrate $10 \mathrm{ml} / \mathrm{Std}$.

Gradientenzentrifugation: $26 \mathrm{ml}$ eines linearen Saccharosegradienten $(20-5 \%)$ in $0,01 \mathrm{M} \mathrm{Na}$-Acetat Puffer, pH 5,1, maschinell gemischt, wełden mit $2 \mathrm{ml}=4$ optische Einheiten Substanzlösung vorsichtig überschichtet und im Schwingrotor SW 25 in einer präparativen Ultrazentrifuge (Beckman L 50) 15 Stdn. bei 25000 U./Min. $=90000 \times g$ (max.)zentrifugiert. Anschließend wird der Gradient von unten abgesaugt und in $2 \mathrm{ml}$ Portionen fraktioniert gesammelt.

Die Einengung der Fraktionen nach Zonenelektrophorese, Gelfiltration und Ultrazentrifugation geschah durch Druckdialyse unter Stickstoff.

Immunelektrophorese wurde in der Modifikation nach SCHEIDEGGER (11) ausgeführt. Die Antisera wurden teils von der Fa. Behring, Marburg bezogen, teils selbst hergestellt.

Analytische Ultrazentrifugation wurde in einer Beckman Zentrifuge Mod. E durchgeführt. Puffer 0,001 M Na-Phosphat, $\mathrm{pH} 7,5+0,2 \mathrm{M}$ $\mathrm{NaCl}$. Die Eiweißkonzentrationen betrugen 0,75-1,5\%. Berechnet wurden die apparenten Sedimentationskoeffizienten $\left(\mathrm{Sc}_{20}\right)$.

Doppeldiffusionstest nach Ouchterlony (10) wurde in Petrischalen von $10 \mathrm{~cm}$ Durchmesser angesetzt. In das zentrale Loch wurde der Antikörper, in die peripheren Löcher die Antigene, Hirnextrakt und die Kontrollantigene, Leber-, Nieren- und Muskelextrakt eingefüllt.

Sämtliche Puffersubstanzen hatten p. a. Qualität.

\section{Ergebnisse}

Die Auftrennung antikörperhaltiger Seren von Patienten mit Multipler Sklerose durch Zonenelektrophorese 
ergab 12 Fraktionen (Abb. 1). Nach der immunelektrophoretischen Analyse der Fraktionen fand sich in Fraktion 1 ausschließlich $\gamma-2$ Globulin, in Fraktion 2 neben $\gamma$-2 auch $\gamma$-1 und $\beta$ Globulin. Fraktion 3 enthielt ferner Haptoglobin und $\alpha-2$ Makroglobulin. Während in Fraktion 4 der $\gamma$-2 Anteil schon fehlt, finden sich hier $\gamma$-1-M, Transferrin, $\beta$-Lipoprotein, $\alpha-2$ Makroglobulin, $\alpha$-Glykoprotein und eine Spur Albumin, ab Fraktion 5 sind $\gamma$-Globuline nicht mehr nachweisbar. Weiter anodisch überwiegt zunehmend das Albumin.

Zur weiteren Reinigung des $\gamma$-1 Makroglobulins werden zunächst die Fraktionen 1 und 2, von denen allerdings nur die Fraktion 2 einen nennenswerten Anteil IgMGlobulin enthält, vereinigt und nach Einengung und Dialyse auf eine Säule mit Sephadex G-200 aufgetragen und mit $0,1 \mathrm{M}$ Tris- $\mathrm{HCl}$ Puffer $+0,2 \mathrm{M} \mathrm{NaCl}$, pH 8,0 eluiert. Das Elutionsdiagramm bei $280 \mathrm{~m} \mu$ zeigte zwei Peaks (Abb. 2).

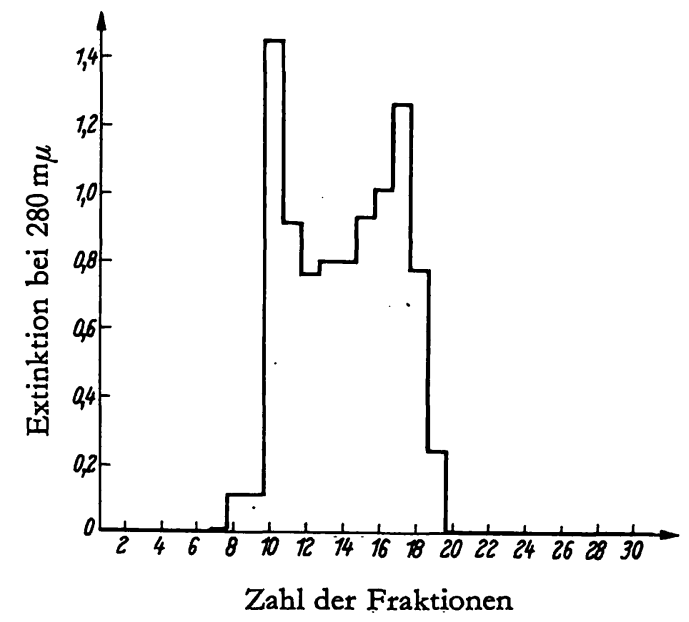

Abb. 1

Zonenelektrophoretische Auftrennung von MS-Serum

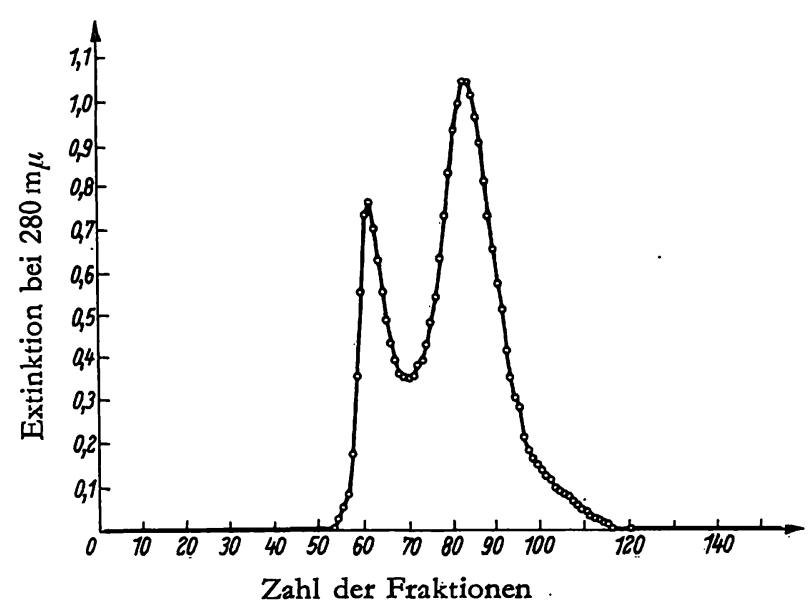

Abb. 2

Auftrennung der vereinigten elektrophoretischen Fraktionen 1 und 2 durch Gelfiltration an Sephadex G-200

Immunelektrophoretisch ergab der erste Peak nur eine Präzipitationslinie, die der Lage nach der $\gamma-1-M$ Linie entsprach, aber etwas weiter kathodisch reichte (Abb. 3). Die Fraktion II nach Gelfiltration enthielt praktisch nur $\gamma-2$ Globulin und eine Spur Transferrin, dagegen kein Makroglobulin. Die Fraktion I nach Gelfiltration wurde anschließend nach Einengung und Dialyse auf einen

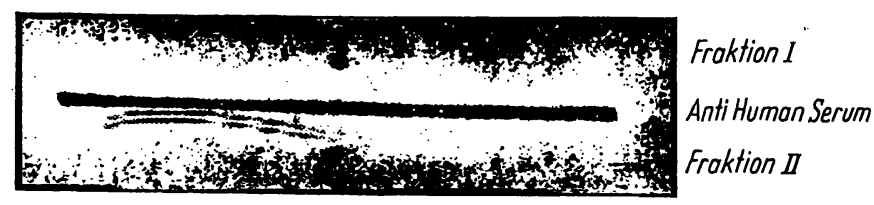

Abb. 3

Immunelektrophoretische Analyse der Fraktionen I und II aus der Gelfiltration

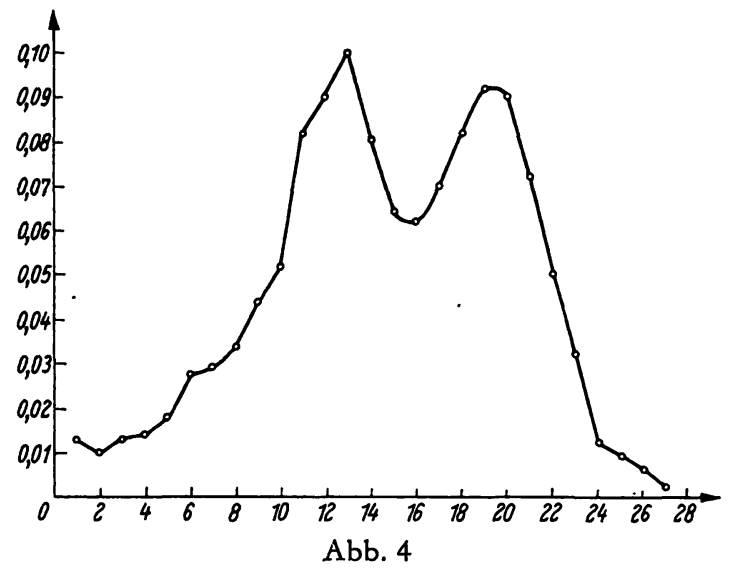

Elutionsdiagramm des Saccharosegradienten, nach Ultrazentrifugation der Fraktion I aus Gelfiltration

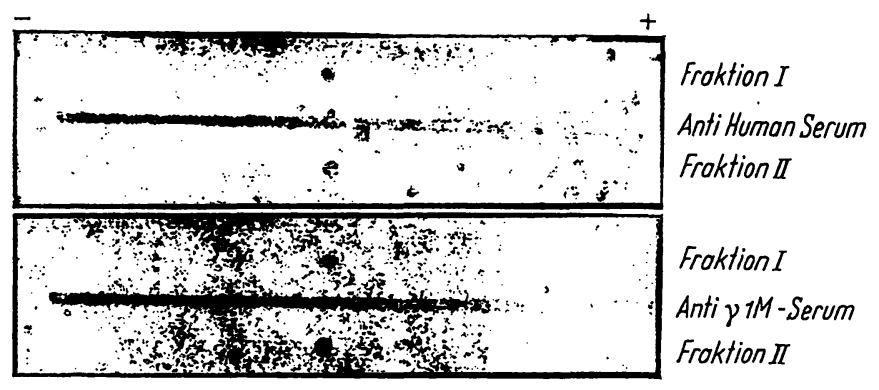

Abb. 5

Immunelektrophoretische Analyse der beiden Fraktionen nach Gradientenzentrifugation

Saccharosegradienten aufgetragen und zentrifugiert. Die Elution des Gradienten ergab eine Extinktionskurve bei $280 \mathrm{~m} \mu$ mit $z$ wei Peaks (Abb. 4).

Die in $2 \mathrm{~m} l$ Portionen gesammelten Fraktionen aus dem Saccharosegradienten wurden vereinigt $z u$ den Fraktionen I und II und zunächst immunelektrophoretisch untersucht. Dabei ergab die Gradientenfraktion I eine typische $\gamma$-1-M Präzipitationslinie, während die Fraktion II eine etwas atypische, weiter kathodisch verlaufende Bande zeigte. Bei Verwendung eines Anti- $\gamma$-1-M Antiserums ließ die Fraktion I eine typische $\gamma$-1-M Bande erkennen, während die Fraktion II keine Reaktion ergab, demnach also keinen $\gamma$-1-M Anteil mehr besaß (Abb. 5). Zur weiteren Charakterisierung der durch verschiedene Trennverfahren isolierten IgMKomponente erfolgte eine analytische Ultrazentrifugation, die für die Fraktion I des Saccharosegradienten einen Sc-Wert von 18,4 ergab.

In der gleichen Weise wie hier beschrieben wurden auch die $\gamma$-1-M-haltigen Zonenelektrophoresefraktionen 3 und 4 weiteren Trennschritten unterworfen. Die Gelfiltration ließ dabei schon ein völlig anderes Verhältnis der Fraktionen zueinander erkennen. Die erste Fraktion war deutlich überwiegend; es fand sich jetzt aber auch 


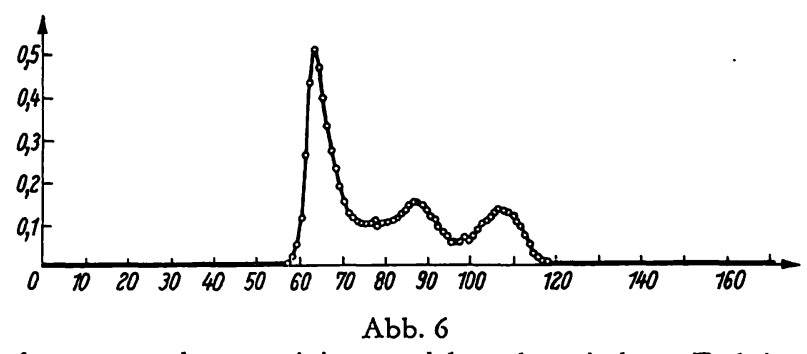

Auftrennung der vereinigten elektrophoretischen Fraktionen 3 und 4 durch Gelfiltration an Sephadex G-200

eine dritte Fraktion, die immunelektrophoretisch Albumin enthielt (Abb. 6). Die immunelektrophoretische Analyse des ersten Peaks der Gelfiltration ergab vier verschiedene Proteine, und zwar neben $\gamma-1-\mathrm{M}$ vor allem $\beta$ - und $\alpha$-Lipoprotein und $\alpha-2$ Makroglobulin. Entsprechend der Immunelektrophorese fanden sich bei der Gradientenzentrifugation vier Peaks, von denen der erste $\gamma-1$ und $\alpha-2$ Makroglobulin enthielt.

Schließlich mußte geprüft werden, ob die isolierten $\alpha-1$ Makroglobuline ihre biologische Aktivität als Antikörper trotz der Anwendung verschiedener Trennverfahren bewahrt hatten. Die Prüfung der $^{\text {"antikörper- }}$ aktivität durch Doppeldiffusion nach OucHTERLONY er-

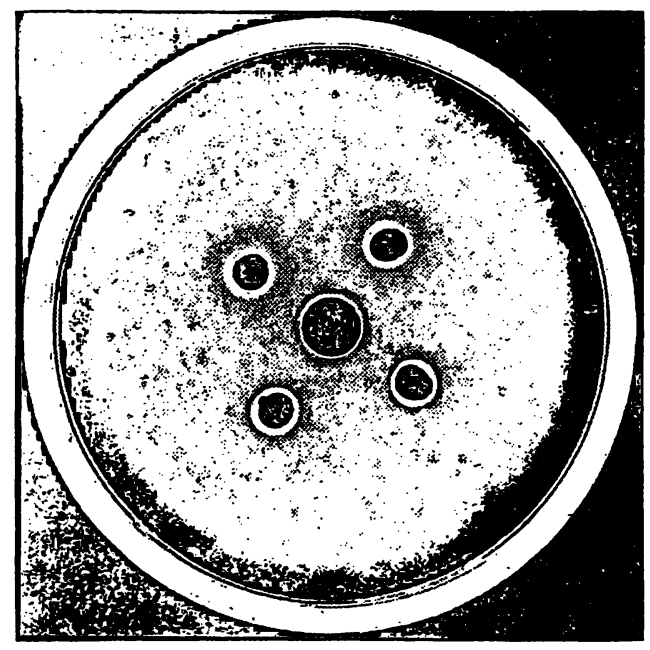

Abb. 7

Nachweis der Antikörpereigenschaft des gereinigten IgM-Globulins durch Doppeldiffusionstest nach OuCHTERLONY zentral: IgM-Globulin (Fraktion I aus Gradient) peripher: Hirnextrakte verschiedener Konzentration gab eindeutige Antikörpereigenschaften der isolierten IgM Globulinfraktion gegen Extrakt aus weißer Hirnsubstanz (Abb. 7).

\section{Diskussion}

Die kombinierte Anwendung von Zonenelektrophorese, Gelfiltration und präparativer Ultrazentrifugation im Saccharosegradienten stellt ein schonendes Verfahren zur Isolierung von Antikörpern von IgM-Typus dar. Wie wir am Beispiel von Autoantikörpern gegen weiße Hirnsubstanz gezeigt haben, bleibt die biologische Aktivität der sehr empfindlichen Antikörper erhalten, d. h. es ist anzunehmen, daß ihre strukturelle Integrität nicht wesentlich beeinträchtigt wurde.

Während die elektrophoretische Trennung von $\gamma$-1 und $\alpha-2$ Makroglobulinen gut gelingt, ist sie weder durch Gelfiltration noch durch Gradientenzentrifugation möglich. Die beiden Moleküle unterscheiden sich wohl in ihrer elektrophoretischen Mobilität, offenbar aber nur wenig nach Größe und Dichte (12). $\gamma$-1 Makroglobuline und $\beta$-Lipoproteine haben demgegenüber ähnliche Ladungsverhältnisse, wie ihre Wanderung im elektrischen Feld zeigt. Auch sind sie wohl wenig unterschiedlich in der Größe, denn durch Gelfiltration allein sind sie nicht zu trennen. Dagegen verhalten sich $\gamma-1$ Makroglobuline und $\beta$-Lipoproteine im Schwerefeld sehr verschieden. Durch Zentrifugation im Saccharosegradienten werden die beiden Proteine vollständig voneinander getrennt. Dieser Befund entspricht Untersuchungen über das Flotationsverhalten von Serumproteinen, nach denen Lipoproteine leichter sind als 1210 , die $\beta$-Lipoproteine sogar leichter als 1019, während die Dichte der meisten nichtfetthaltigen Plasmaproteine zwischen 1260 und 1380 liegt $(13,14,15)$.

Zur Isolierung eines reinen IgM-Globulins ist eine Kombination mehrerer Trennverfahren notwendig. Die vọn uns angewendete Kombination, nach der Proteine nach dem isoelektrischen Punkt, nach der Größe und nach der Dichte getrennt werden, erwies sich sowohl hinsichtlich des Trenneffektes als auch in bezug auf die Erhaltung der biologischen Aktivität der Antikörper als brauchbar. Bei sehr günstigen Ausgangsbedingungen und geringerer Anforderung an den Reinheitsgrad wird man u. U. auf die Molekularsiebung verzichten können.

\section{Literatur}

1. World Health Organization Committee on Nomenclature for Human Immunoglobulins. Bull. World Health Organizat., N. Y. 30, 447 (1964). - 2. Rowe, D. S. und J. L. FAHEY, J. exp. Med. 121, 171 (1965). - 3. Heide, K. und H. Haupt, Behringwerk-Mitt., Marburg 43, 161 (1964). - 4. MüLlER-EbERhard, H. J., H. G. Kunkel und E. C. Franklin, Proc. Soc. exp. Biol. Med. 93, 146 (1956). - 5. Kunkel, H. G. in: F. W. Putnam, The Plasma Proteins I, S. 294, Academic Press, New York (1960). - 6. Vaerman, J. P., J. F. Heremans und C. Vaerman, J. Immunology 91, 7 (1963). - 7. ReISNer, C. A. und E. C. FrankLIN, J. Immunology 87, 654 (1961). - 8. Walton, P. W., D. S. Rowe,
J. F. Soothill und D. R. Stanworth, Immunology 6, 305 (1963). - 9. Ross, J., K. Schumacher und H. Mengete, Klin. Wschr. 43, 1324 (1965). - 10. Ross, J., Verh. Dtsch. Ges. inn. Med. 68, 566 (1962). - 11. ScheidegGer, J. J., Internat. Arch. Allergy, Basel 7, 103 (1955). - 12. Deutsch, H. F. und J. I. Morton, J. biol. Chemistry 231, 1107 (1958). - 13. Wuhrmann, F. und H. H. Märkr, Dysproteinämien und Paraproteinämien, Benno Schwabe \& Co., Basel-Stuttgart (1963). - 14. Pezold, F. A., Verh. Dtsch. Ges. inn. Med. 66, 427 (1960). - 15. Pezord, F. A., Lipide und Lipoproteide im Blutplasma, Springer-Verlag, Berlin-Göttingen-Heidelberg (1961).

Dr. K. Schumacher

Med. Univ.-Klinik, 5 Köln-Lindenthal, Lindenburg 